\title{
A HEURISTIC ALGORITHM FOR THE DETERMINISTIC MULTI-PRODUCT INVENTORY SYSTEM WITH CAPACITY CONSTRAINT
}

\author{
Maw-Sheng Chern Kao-Chêng Lin Chin-Chern Chen \\ National Tsing Hua University
}

(Received March 22, 1988; Final November 26, 1990)

\begin{abstract}
This paper investigates an $m$-product inventory system $(m \geq 3)$ with a capacity constraint where products can have individual order intervals and orders be phased to reduce the maximum stock level of all the products on hand. The objective is then to find the optimal order quantity of each product by considering staggering time and order interval which minimizes the system cost per unit time. The problem is described in a non-linear integer programming problem which shows a very complicated nature to derive the solution analytically. Therefore, a heuristic algorithm is proposed and tested for its efficiency with various numerical examples as being superior to either the Lagrangian multiplier method or the fixed cycle method.
\end{abstract}

\section{Introduction}

This paper considers a multi-product inventory ordering problem of minimizing the average inventory cost subject to a capacity constraint. The Lagrangian multiplier method [3, $7]$ and fixed cycle method $[2,6,8,10]$ have been used for solving such a problem. However, the Lagrangian multiplier method implicitly assumes that all products will be ordered simultaneously, or at least within an arbitrarily small time interval. Thus, the warehouse space utilization is about $50 \%$ and it usually results in higher average cost per unit time. Although the fixed cycle method allows the phasing of orders for the different products, it assumes that all products have the same order interval. In some cases, this restriction may lead to a higher average cost per unit time than that of the Lagrangian multiplier method. Hartley and Thomas [4,9] and Chen et al. [1] propose an alternative approach in which different products may have different order intervals and these orders may be phased in certain cases to avoid having the maximum stock levels of different products on hand at the same time (see, for example, Figure 2). For the two - product problem we may assume without loss of generality that one of the staggering times is zero and the maximum stock level over time can be expressed explicitly as a function of the order intervals and the nonzero staggering time $[4,9]$. Moreover, we can minimize the maximum stock level with respect to the staggering time and express it as a function of the order intervals. The average cost per unit time can then be minimized by using such an expression.

However, for $m$-product problems, $m \geq 3$, it is difficult to derive a similar relationship among the maximum stock level, order intervals and staggering times. The problem rather reveals so complex nature that it is hard to solve. Therefore, this paper proposes a heuristic algorithm which is tested with various numerical examples for its superiority to the Lagrangian multiplier method and the fixed cycle method. It is shown that the proposed algorithm gives better solutions than the latter two methods. 


\section{Mathematical Formulation}

Assumptions:

1. Demand can be approximated by a constant average demand over time.

2. Planning horizon is infinite.

3. Shortage is not allowed.

4. Delivery is instantaneously made.

5. Overall stock level is periodic.

\section{Notation:}

$c_{i}=$ the order cost of product $i$. (Assuming that the order cost is independent of the order quantity.)

$v_{i}=$ the volume of product $i$ per unit.

$d_{i}=$ the demand of product $i$ per unit time.

$h_{i}=$ the holding cost of one unit of product $i$ per unit time.

$q_{i}=$ the order quantity of product $i$.

$t_{i}=$ the order interval of product $i .\left(q_{i}=d_{i} t_{i}.\right)$

$s_{i}=$ the staggering time of product $i$.

$M=$ the warehouse capacity.

The total variable cost is the sum of order costs and holding costs. For given $c_{i}, v_{i}, d_{i}, h_{i}$, $i=1, \cdots, m$, and $M$, we let $Z$ be the total variable cost per unit time and $S_{\max }(\mathbf{t}, \mathbf{s})$ be the maximum stock level. Then, the problem is mathematically stated as follows:

$$
\begin{aligned}
& \operatorname{minimize} Z=\sum_{i=1}^{m} \frac{c_{i}}{t_{i}}+0.5 \sum_{i=1}^{m} h_{i} d_{i} t_{i} \\
& \text { subject to } S_{\max }(\mathbf{t}, \mathbf{s}) \leq M, s_{i} \geq 0, t_{i}>0, i=1, \cdots, m,
\end{aligned}
$$

where $\mathbf{t}=\left(t_{1}, \cdots, t_{m}\right)$ and $\mathbf{s}=\left(s_{1}, \cdots, s_{m}\right)$. The objective is to find $(\mathbf{t}, \mathbf{s})$ that minimizes $Z$ and satisfies the constraint (2).

\section{Staggering the Initial Orders}

Let $\left\lfloor x_{0}\right\rfloor$ denote the largest integer which is smaller than or equal to $x_{0}$. Then, given the order interval $t_{i}$ and the staggering times $s_{i}, 0 \leq s_{i}<t_{i}, i=1, \cdots, m$, the stock volume of product $i$ is

$$
f_{i}(t)=v_{i} d_{i} t_{i}+v_{i} d_{i} s_{i}-v_{i} d_{i}\left(t-\left\lfloor\frac{t-s_{i}}{t_{i}}\right\rfloor t_{i}\right)
$$

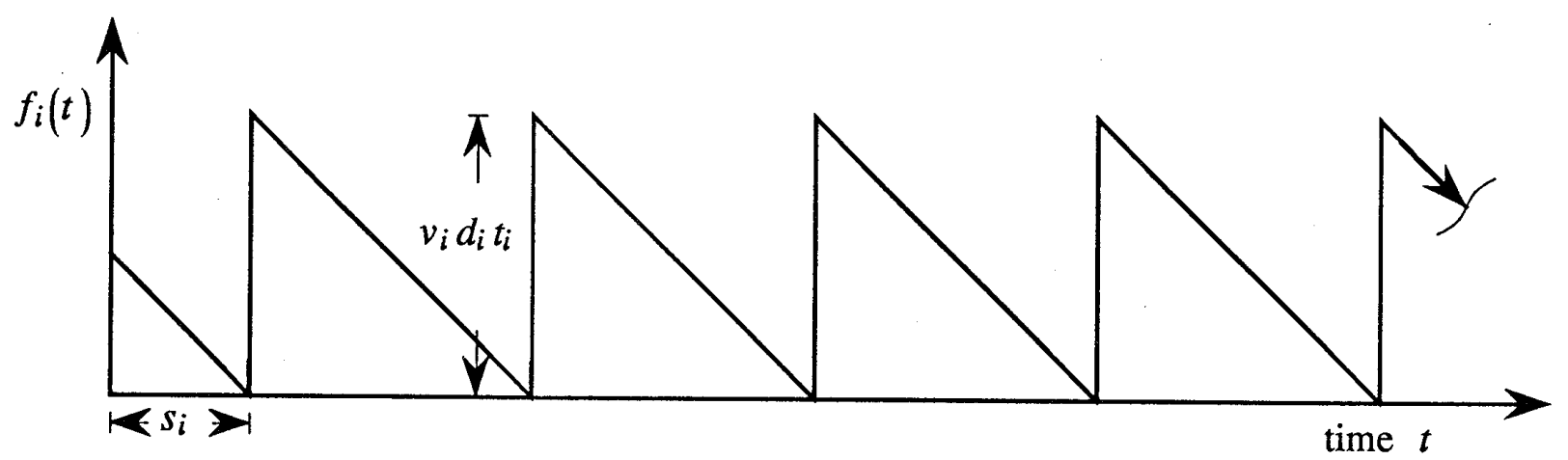

Figure 1. Stock level of product $i$. 


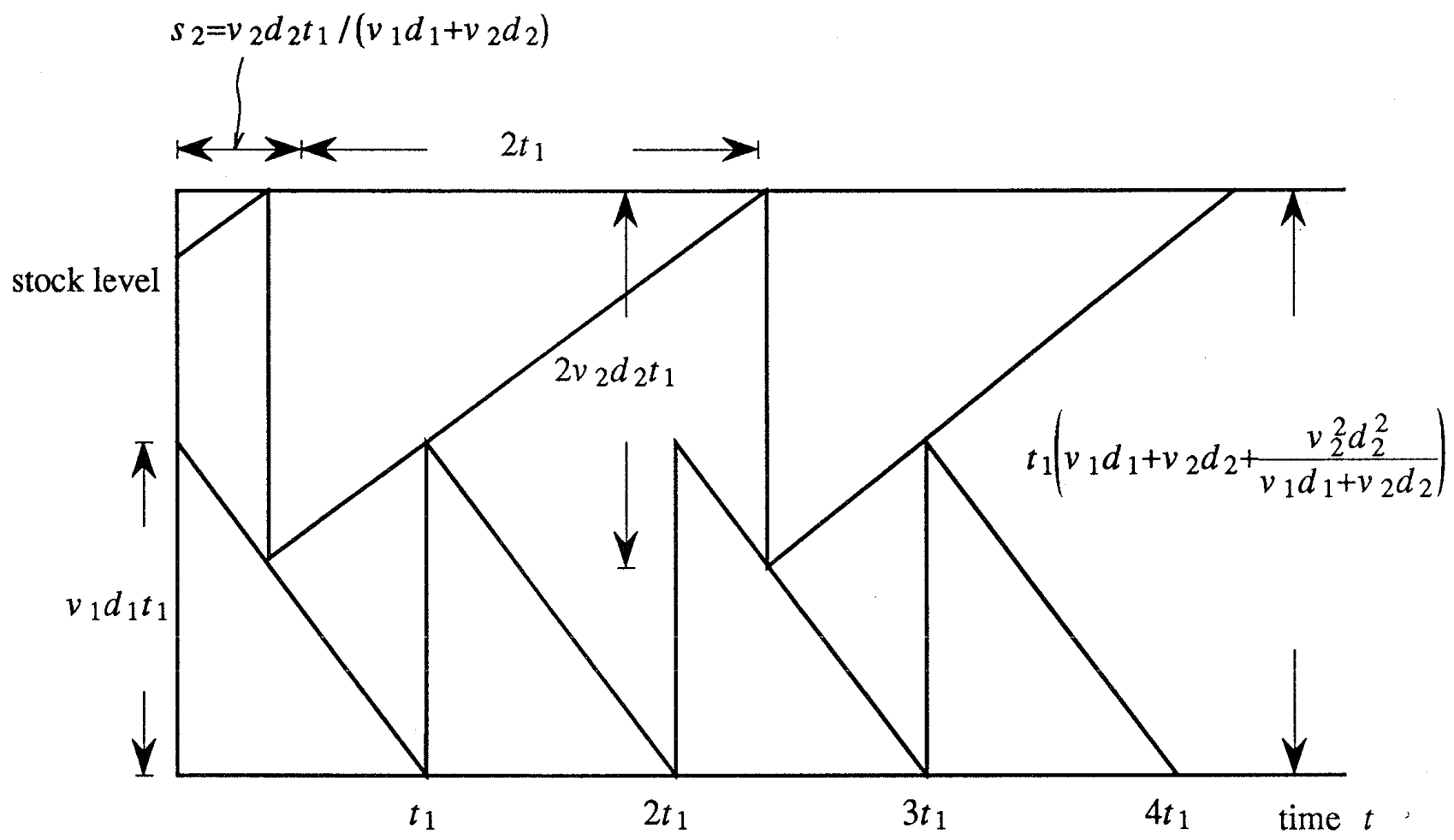

Figure 2. Stock level over time with $t_{2}=2 t_{1}[2]$.

If $t_{i}, i=1, \cdots, m$, are rational numbers, then $f_{i}(t)$ is periodic with the period $t_{i}$ and $\sum_{i=1}^{m} f_{i}(t)$ is also periodic with the period $T=\min \left\{t \mid t\right.$ is an integral multiple of $t_{i}, i=$ $1, \cdots, m\}$.

Theorem 1. Let $t_{i}, i=1, \cdots, m$, be the period of $f_{i}(t)$. Then

$$
\begin{gathered}
S_{\max }(\mathbf{t}, \mathbf{s})=\max \left\{\sum_{i=1}^{m} f_{i}(t) \mid 0 \leq t \leq T\right\} \\
=\sum_{i=1}^{m} v_{i} d_{i} t_{i}-\min \left\{S_{k}\left(s_{1}, \cdots, s_{m}\right) \mid k=1, \cdots, m\right\} \text {, where } \\
S_{k}\left(s_{1}, \cdots, s_{m}\right)=\min _{\ell_{k k}, \ell_{k i}}\left\{\sum_{i \neq k} v_{i} d_{i}\left(s_{k}+\ell_{k k} t_{k}-s_{i}-\ell_{k i} t_{i}\right) \mid \ell_{k i} \in\left[0, n_{i}-1\right], \ell_{k k} \in\left[0, n_{k}-1\right],\right. \\
\left.n_{i}=T / t_{i}, \ell_{k i}, \ell_{k k} \text { integer and } s_{k}+\ell_{k k} t_{k}-s_{i}-\ell_{k i} t_{i} \geq 0 \text { for each } i .\right\} .
\end{gathered}
$$

Proof. It is clear that the maximum stock level will be attained immediately after one of the $m$ products is ordered. Without loss of generality, we assume that the maximum stock level is attained at $t=\eta_{k}$ when the product $k$ is ordered. 
Let $\eta_{k}-s_{k}=\underline{\ell}_{k k} t_{k}$ where $\underline{\ell}_{k k}=\left\lfloor\left(\eta_{k}-s_{k}\right) / t_{k}\right\rfloor$ is a nonnegative integer.

$$
\begin{aligned}
\sum_{i=1}^{m} f_{i}\left(\eta_{k}\right) & =\sum_{i=1}^{m}\left[v_{i} d_{i} t_{i}+v_{i} d_{i} s_{i}-v_{i} d_{i}\left(\eta_{k}-\left\lfloor\left(\eta_{k}-s_{i}\right) / t_{i}\right\rfloor t_{i}\right)\right] \\
& =\sum_{i=1}^{m} v_{i} d_{i} t_{i}-\sum_{i \neq k} v_{i} d_{i}\left(\eta_{k}-s_{i}-\left\lfloor\left(\eta_{k}-s_{i}\right) / t_{i}\right\rfloor t_{i}\right) \\
& =\sum_{i=1}^{m} v_{i} d_{i} t_{i}-\sum_{i \neq k} v_{i} d_{i}\left(s_{k}+\underline{\ell}_{k k} t_{k}-s_{i}-\underline{\ell}_{k i} t_{i}\right)
\end{aligned}
$$

(where $\underline{\ell}_{k i}=\left\lfloor\left(s_{k}+\underline{\ell}_{k k} t_{k}-s_{i}\right) / t_{i}\right\rfloor$ and $\underline{\ell}_{k i} \in\left[0, n_{i}-1\right]$ for each $i$.)

$$
\begin{aligned}
= & \sum_{i=1}^{m} v_{i} d_{i} t_{i}-\min _{\left(\ell_{k i}\right)}\left\{\sum_{i \neq k} v_{i} d_{i}\left(s_{k}+\underline{\ell}_{k k} t_{k}-s_{i}-\ell_{k i} t_{i}\right) \mid \ell_{k i} \in\left[0, n_{i}-1\right], \ell_{k i}\right. \text { integer, } \\
& \left.s_{k}+\underline{\ell}_{k k} t_{k}-s_{i}-\ell_{k i} t_{i} \geq 0 \text { and } n_{i}=T / t_{i} \text { for each } i .\right\} \\
= & \sum_{i=1}^{m} v_{i} d_{i} t_{i}-S_{k}\left(s_{1}, \cdots, s_{m}\right) .
\end{aligned}
$$

Hence, the proof is completed.

By Theorem 1, we can also formulate the problem (1) - (2) as a mixed-integer programming problem as follows:

$$
\begin{aligned}
& \operatorname{minimize} Z=\sum_{i=1}^{m} \frac{c_{i}}{t_{i}}+0.5 \sum_{i=1}^{m} h_{i} d_{i} t_{i}, \\
& \text { subject to } \sum_{i=1}^{m} v_{i} d_{i} t_{i}-\sum_{i \neq k} v_{i} d_{i}\left(s_{k}+\ell_{k k} t_{k}-s_{i}-\ell_{k i} t_{i}\right) \leq M, k=1, \cdots, m, \\
& \quad s_{k}+\ell_{k k} t_{k}-s_{i}-\ell_{k i} t_{i} \geq 0, i=1, \cdots, m, k=1, \cdots, m, i \neq k, \\
& \quad 0 \leq \ell_{k i} \leq n_{i}-1, n_{i}=\frac{T}{t_{i}}, i=1, \cdots, m, \\
& \\
& s_{i} \geq 0, T>0, t_{i}>0, \ell_{k i} \text { integer, } i=1, \cdots, m, k=1, \cdots, m .
\end{aligned}
$$

Although a branch and bound method [5] can be designed for solving (3) - (7), the complexity of constraints reveals that it may take a large amount of computational time to solve this problem even when $m=3$, because for some problem instances a large number of $\ell_{k i}$ 's must be enumerated. Therefore, some approaches have been proposed for solutions in the subspaces of (4) - (7). For example, the Lagrangian multiplier method searches for the optimal solution in the subspace of $(4)-(7)$ with $s_{1}=s_{2}=\cdots=s_{m}=0$ and the fixed cycle method searches for the optimal solution in the subspace with $t_{1}=t_{2}=\cdots=t_{m}$. In this paper, however, we propose an algorithm which searches for a solution of $(3)-(7)$ such that $s_{i}$ and $t_{i}, i=1, \cdots, m$ satisfy some specific relationships.

For $m=2$, we may assume without loss or generality that $s_{1}=0$. By Theorem 1 ,

$$
\max _{0 \leq t \leq T}\left\{f_{1}(t)+f_{2}(t)\right\}=v_{1} d_{1} t_{1}+v_{2} d_{2} t_{2}-\min \left\{S_{1}\left(0, s_{2}\right), S_{2}\left(0, s_{2}\right)\right\}
$$

It has been shown [4] that for given $t_{1}$ and $t_{2}$, the optimal solution of (8) satisfies the following condition

$$
s_{2}=\frac{T}{n_{1} n_{2}} \frac{v_{2} d_{2}}{v_{1} d_{1}+v_{2} d_{2}}
$$


and the maximum stock level is

$$
\max _{0 \leq t \leq T}\left\{f_{1}(t)+f_{2}(t)\right\}=v_{1} d_{1} t_{1}+v_{2} d_{2} t_{2}-\frac{T}{n_{1} n_{2}} \frac{v_{1} d_{1} v_{2} d_{2}}{v_{1} d_{1}+v_{2} d_{2}}
$$

In this case, the optimal solution of $(1)-(2)$ can then be solved by using (9) - (10) [9].

For $m \geq 3$, it is difficult to derive a similar relationship among the optimal staggering times $s_{i}$ and the order intervals $t_{i}, i=1, \cdots, m$, as that in (9). Therefore, we propose a heuristic approach for scheduling the initial orders. We denote that

$$
T_{i j}=T_{j i}=n_{i j} t_{i}=n_{j i} t_{j}=\min \left\{t \mid t \text { is an integral multiple of } t_{i} \text { and } t_{j} .\right\}
$$

where $n_{i j}$ and $n_{j i}$ are co-prime, i.e. $\operatorname{gcd}\left(n_{i j}, n_{j i}\right)=1$. For given $k$ and $\left(r_{i}\right)_{i \neq k}, \sum_{i \neq k} r_{i}=1$, we consider product $i$ and $r_{i}$ proportion of product $k$ as a two-product problem and let the staggering times be of the form as that in (9). Therefore, if we let

$$
s_{i}=\frac{T_{k i}}{n_{k i} n_{i k}} \frac{v_{i} d_{i}}{r_{i} v_{k} d_{k}+v_{i} d_{i}}, i=1, \cdots, m, i \neq k
$$

and $s_{k}=0$, then by $(9)-(10)$ the maximum stock level is not greater than

$$
\min \left\{\sum_{i=1}^{m} v_{i} d_{i} t_{i}-\sum_{i \neq k} \frac{T_{k i}}{n_{k i} n_{i k}} \frac{r_{i} v_{k} d_{k} v_{i} d_{i}}{r_{i} v_{k} d_{k}+v_{i} d_{i}} \mid \sum_{i \neq k} r_{i}=1, r_{i} \geq 0 \text { for each } i .\right\}
$$

When $m=2,(11)$ is reduced to (9) and (12) is reduced to (10) with $k=1, s_{1}=$ $0, r_{1}=0, n_{12}=n_{1}$ and $n_{21}=n_{2}$. Moreover, in Figure 3 and Figure 4, we consider a three-product problem. For fixed $r$, if we let the staggering times be $s_{1}=0, s_{2}=$ $\left(T_{12} / n_{12} n_{21}\right)\left[v_{2} d_{2} /\left(r v_{1} d_{1}+v_{2} d_{2}\right)\right]$ and $s_{3}=\left(T_{13} / n_{13} n_{31}\right)\left[v_{3} d_{3} /\left((1-r) v_{1} d_{1}+v_{3} d_{3}\right)\right]$, then the stock volume of product 2 with $r 100 \%$ proportion of product 1 is not greater than

$$
r v_{1} d_{1} t_{1}+v_{2} d_{2} t_{2}-\frac{T_{12}}{n_{12} n_{21}} \frac{r v_{1} d_{1} v_{2} d_{2}}{r v_{1} d_{1}+v_{2} d_{2}}
$$

and the stock volume of product 3 with $(1-r) 100 \%$ proportion of product 1 is not greater than

$$
(1-r) v_{1} d_{1} t_{1}+v_{3} d_{3} t_{3}-\frac{T_{13}}{n_{13} n_{31}} \frac{(1-r) v_{1} d_{1} v_{3} d_{3}}{(1-r) v_{1} d_{1}+v_{3} d_{3}}
$$




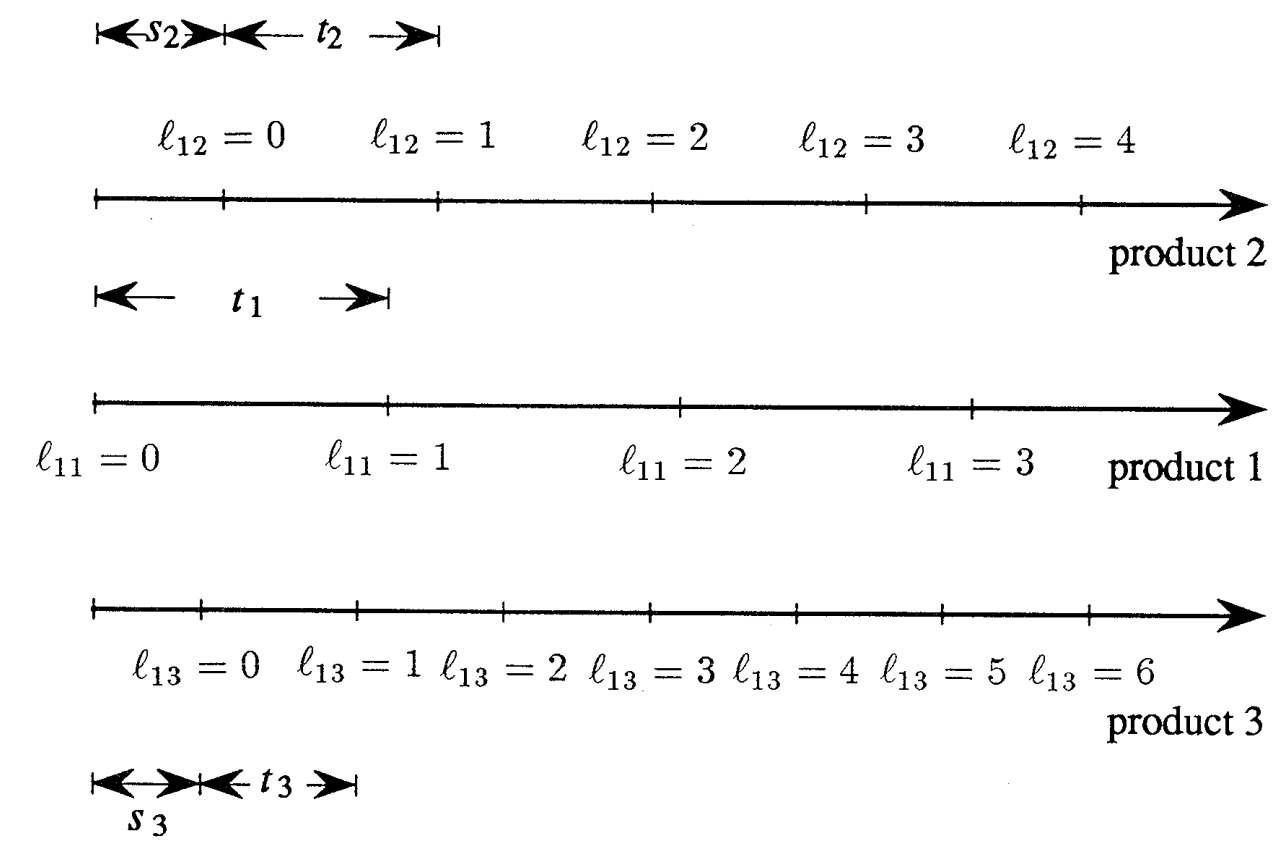

Figure 3. Staggering times and order intervals of three products.

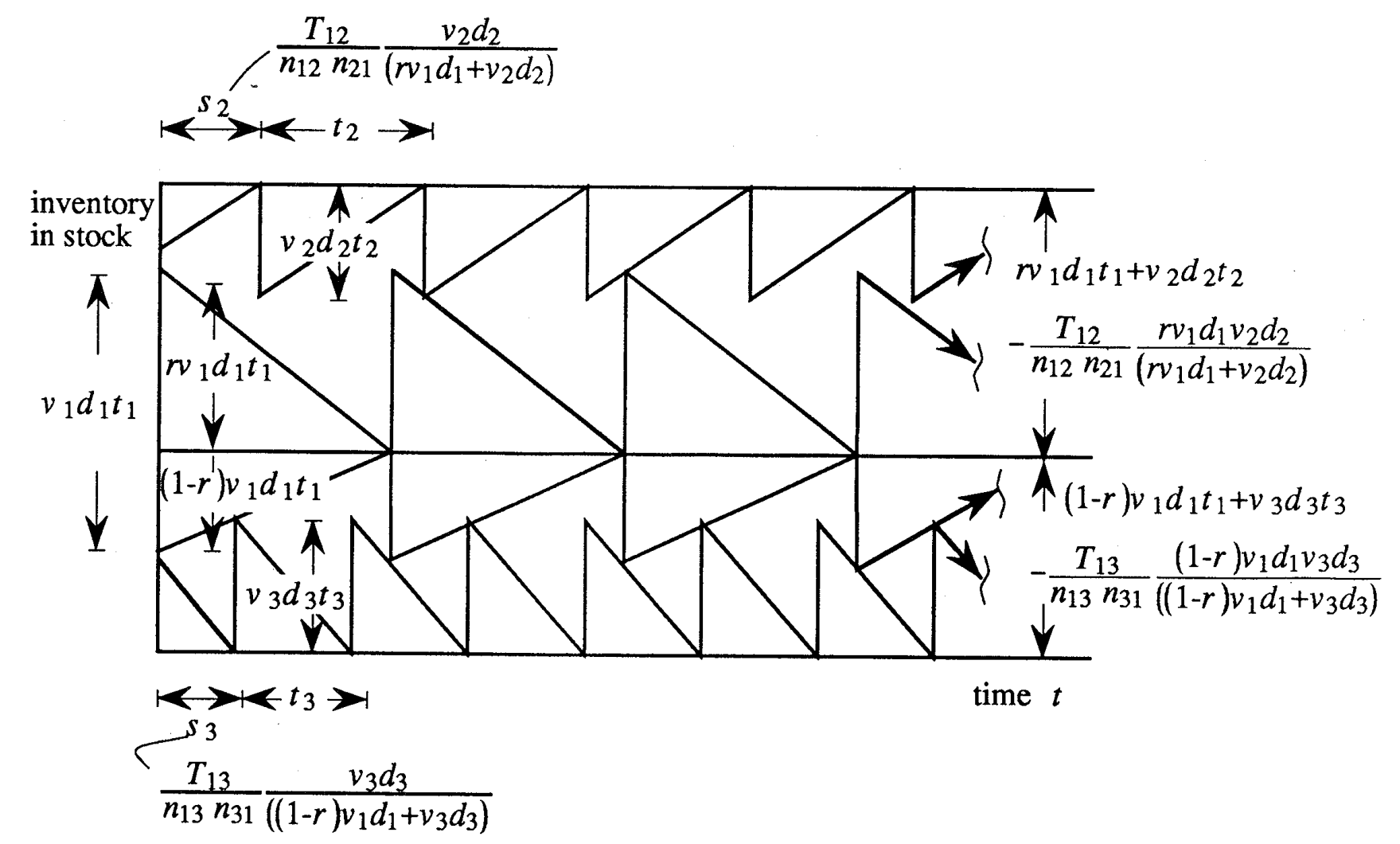

Figure 4. Stock level of three-product inventory over time.

For each $k, 1 \leq k \leq m$, we consider a simplified problem which is obtained from (1)(2) by replacing (2) with an approximation of capacity constraint by using (12).

$$
\operatorname{minimize} \sum_{i=1}^{m} \frac{c_{i}}{t_{i}}+0.5 \sum_{i=1}^{m} h_{i} d_{i} t_{i}
$$




$$
\begin{gathered}
\text { subject to } \sum_{i=1}^{m} v_{i} d_{i} t_{i}-\sum_{i \neq k} \frac{T_{k i}}{n_{k i} n_{i k}} \frac{r_{i} v_{k} d_{k} v_{i} d_{i}}{r_{i} v_{k} d_{k}+v_{i} d_{i}} \leq M, \\
T_{i j}=T_{j i}=n_{i j} t_{i}=n_{j i} t_{j}=\min \left\{t \mid t \text { is an integral multiple of } t_{i} \text { and } t_{j}\right\}, \\
\sum_{i \neq k} r_{i}=1, r_{i} \geq 0, t_{i}>0, n_{i k}>0, n_{k i}>0, n_{i k}, n_{k i} \text { integer. }
\end{gathered}
$$

For each $k$, we will solve (13) - (16) for an approximate solution of $(1)-(2)$. We then select the best one of these $m$ solutions. In the following sections, a procedure is proposed for solving (13) - (16).

\section{A Heuristic Method}

For given $k,(13)-(16)$ can be formulated as follows:

$$
\begin{aligned}
& \operatorname{minimize} W=\left(c_{k}+\sum_{i \neq k} \frac{n_{i k}}{n_{k i}} c_{i}\right) \frac{1}{t_{k}}+0.5\left(h_{k} d_{k}+\sum_{i \neq k} \frac{n_{k i}}{n_{i k}} h_{i} d_{i}\right) t_{k}, \\
& \text { subject to }\left(v_{k} d_{k}+\sum_{i \neq k} \frac{n_{k i}}{n_{i k}} v_{i} d_{i}-\sum_{i \neq k} \frac{1}{n_{i k}} \frac{r_{i} v_{k} d_{k} v_{i} d_{i}}{r_{i} v_{k} d_{k}+v_{i} d_{i}}\right) t_{k} \leq M, \\
& \sum_{i \neq k} r_{i}=1, r_{i} \geq 0, t_{k}>0, n_{i k}>0, n_{k i}>0, n_{i k}, n_{k i} \text { integer, }
\end{aligned}
$$

where (17) and (18) are derived from (13) and (14) respectively. For given $\left(n_{i k}\right)_{i \neq k}=$ $\left(\underline{n}_{i k}\right)_{i \neq k}$, we can further convert this mixed-integer programming problem into a simplified problem by minimizing the objective function $W$ with respect to $t_{k}$. Note that $W$ is a convex function of $t_{k}$. For $i \neq k$, let $a_{i}=n_{k i} / \underline{n}_{i k}$. The solution of $\partial W / \partial t_{k}=0$ is

$$
\underline{t}_{k}=\sqrt{2\left(c_{k}+\sum_{i \neq k} \frac{c_{i}}{a_{i}}\right) /\left(h_{k} d_{k}+\sum_{i \neq k} a_{i} h_{i} d_{i}\right)}
$$

Substituting $t_{k}$ by $\underline{t}_{k}$ in $(17)$, we have

$$
W=\Phi\left(a_{1}, \cdots, a_{k-1}, a_{k+1}, \cdots, a_{m}\right)=\sqrt{2\left(c_{k}+\sum_{i \neq k} \frac{c_{i}}{a_{i}}\right)\left(h_{k} d_{k}+\sum_{i \neq k} a_{i} h_{i} d_{i}\right)} .
$$

Consider the problem

$$
\operatorname{minimize}_{a_{i} \geq 0} \Phi\left(a_{1}, \cdots, a_{k-1}, a_{k+1}, \cdots, a_{m}\right)
$$

and let $\left(a_{i}^{*}\right)_{i \neq k}$ be an optimal solution of $(20)$. Because $W$ is a convex function of $\left(n_{k i}\right)_{i \neq k}$, the optimal solution $\left(n_{k i}\right)_{i \neq k}$ associated with $\left(a_{i}^{*}\right)_{i \neq k}$ would be $n_{k i}=\left\lfloor\underline{n}_{i k} a_{i}^{*}\right\rfloor$ or $\left\lfloor\underline{n}_{i k} a_{i}^{*}\right\rfloor+1$ for each $i \neq k$. For given $\left(n_{k i}\right)_{i \neq k}$ where $n_{k i}=\left\lfloor\underline{n}_{i k} a_{i}^{*}\right\rfloor$ or $\left\lfloor\underline{n}_{i k} a_{i}^{*}\right\rfloor+1$, we then search for an optimal solution $\left(r_{i}^{*}\right)_{i \neq k}$ which minimizes the left-hand side of (18) with $t_{k}=\underline{t}_{k}$ and $\left(n_{i k}\right)_{i \neq k}=\left(\underline{n}_{i k}\right)_{i \neq k}$.

Thus, if

$$
\left(v_{k} d_{k}+\sum_{i \neq k} a_{i}^{*} v_{i} d_{i}-\sum_{i \neq k} \frac{1}{\underline{n}_{i k}} \frac{r_{i}^{*} v_{k} d_{k} v_{i} d_{i}}{r_{i}^{*} v_{k} d_{k}+v_{i} d_{i}}\right) \underline{t}_{k} \leq M
$$

then

$$
\min \left\{\Phi \mid n_{k i}=\left\lfloor\underline{n}_{i k} a_{i}^{*}\right\rfloor,\left\lfloor\underline{n}_{i k} a_{i}^{*}\right\rfloor+1, i=1, \cdots, m, i \neq k,\right.
$$




$$
\left.\left(v_{k} d_{k}+\sum_{i \neq k} \frac{n_{k i}}{\underline{n}_{i k}} v_{i} d_{i}-\sum_{i \neq k} \frac{1}{\underline{n}_{i k}} \frac{r_{i}^{*} v_{k} d_{k} v_{i} d_{i}}{r_{i}^{*} v_{k} d_{k}+v_{i} d_{i}}\right) \underline{t}_{k} \leq M\right\}
$$

yields an optimal solution for $(17)-(19)$ with $t_{k}=\underline{t}_{k}$ and $\left(n_{i k}\right)_{i \neq k}=\left(\underline{n}_{i k}\right)_{i \neq k}$. Otherwise, by the property of complementary slackness, the capacity constraint (18) is tight. If the capacity constraint is tight, we let $\underline{t}_{k}^{\prime}=M / \xi$ where

$$
\xi=v_{k} d_{k}+\sum_{i \neq k} a_{i} v_{i} d_{i}-\sum_{i \neq k} \frac{1}{n_{i k}} \frac{r_{i} v_{k} d_{k} v_{i} d_{i}}{r_{i} v_{k} d_{k}+v_{i} d_{i}}
$$

Substituting $t_{k}$ by $\underline{t}_{k}^{\prime}$ in (17), we have

$$
\begin{aligned}
W & =\Psi\left(a_{1}, \cdots, a_{k-1}, a_{k+1}, \cdots, a_{m}, r_{1}, \cdots, r_{k-1}, r_{k+1}, \cdots, r_{m}\right) \\
& =\left(c_{k}+\sum_{i \neq k} \frac{c_{i}}{a_{i}}\right) \frac{\xi}{M}+0.5\left(h_{k} d_{k}+\sum_{i \neq k} a_{i} h_{i} d_{i}\right) \frac{M}{\xi} .
\end{aligned}
$$

Then, (17) - (19) can be written as follows:

$$
\begin{aligned}
& \operatorname{minimize} \Psi\left(a_{1}, \cdots, a_{k-1}, a_{k+1}, \cdots, a_{m}, r_{1}, \cdots, r_{k-1}, r_{k+1}, \cdots, r_{m}\right), \\
& \text { subject to } \sum_{i \neq k} r_{i}=1, r_{i} \geq 0, a_{i} \geq 0, \text { for all } i .
\end{aligned}
$$

Similarly, we solve $(22)-(23)$ for a solution $\left(a_{i}^{*}, r_{i}^{*}\right)_{i \neq k}$.

Hence, for given $\left(\underline{n}_{i k}\right)_{i \neq k}$ and $\left(a_{i}^{*}\right)_{i \neq k}$, if the capacity constraint $(21)$ is satisfied with $\left(r_{i}\right)_{i \neq k}=\left(r_{i}^{*}\right)_{i \neq k}$, then an approximate solution of $(13)-(16)$ can be obtained by solving

$$
\begin{gathered}
\min \left\{\Phi \mid n_{k i}=\left\lfloor\underline{n}_{i k} a_{i}^{*}\right\rfloor,\left\lfloor\underline{n}_{i k} a_{i}^{*}\right\rfloor+1, i=1, \cdots, m, i \neq k,\right. \\
\left.\left(v_{k} d_{k}+\sum_{i \neq k} \frac{n_{k i}}{\underline{n}_{i k}} v_{i} d_{i}-\sum_{i \neq k} \frac{1}{\underline{n}_{i k}} \frac{r_{i}^{*} v_{k} d_{k} v_{i} d_{i}}{r_{i}^{*} v_{k} d_{k}+v_{i} d_{i}}\right) \underline{t}_{k} \leq M\right\} .
\end{gathered}
$$

Otherwise, a solution is obtained by solving

$$
\begin{gathered}
\min \left\{\Psi \mid n_{k i}=\left\lfloor\underline{n}_{i k} a_{i}^{*}\right\rfloor,\left\lfloor\underline{n}_{i k} a_{i}^{*}\right\rfloor+1, i=1, \cdots, m, i \neq k,\right. \\
\left.\left(v_{k} d_{k}+\sum_{i \neq k} \frac{n_{k i}}{\underline{n}_{i k}} v_{i} d_{i}-\sum_{i \neq k} \frac{1}{\underline{n}_{i k}} \frac{r_{i}^{*} v_{k} d_{k} v_{i} d_{i}}{r_{i}^{*} v_{k} d_{k}+v_{i} d_{i}}\right) \underline{t}_{k} \leq M\right\} .
\end{gathered}
$$

We conclude that a solution $\left(t_{i}^{*}\right)$ of $(13)$ - (16) can be obtained by solving either (24) or (25) for all possible values of $\underline{n}_{i k}$. In order to solve (24) or (25) efficiently, we will derive an upper bound of $n_{i k}$ for each $i \neq k$ in the next section.

\section{An Upper Bound of $n_{i k}$}

Let

$$
\mu(B)=\min \left\{\sum_{i=1}^{m} \frac{c_{i}}{t_{i}}+0.5 \sum_{i=1}^{m} h_{i} d_{i} t_{i} \mid \sum_{i=1}^{m} v_{i} d_{i} t_{i} \leq M+B, t_{i}>0, i=1, \cdots, m\right\} .
$$

If $B=0$, then $\mu(0)$ is a convex program which can be solved by the Lagrangian multiplier method [3]. Assume that $\lambda^{*}$ is an optimal multiplier of (26) with $B=0$. Then

$$
\mu(B) \geq \mu(0)-\lambda^{*} B \text { for any } B \geq 0[9] .
$$


For given $k$, let

$$
B_{k}=\sum_{i \neq k} \frac{t_{k}}{n_{i k}} \frac{r_{i} v_{k} d_{k} v_{i} d_{i}}{r_{i} v_{k} d_{k}+v_{i} d_{i}}
$$

and $U$ be the objective value associated with a feasible solution of (1) - (2) such that $\mu\left(B_{k}\right) \leq U \leq \mu(0)$. Then an upper bound $u_{i k}$ of $n_{i k}$ can be derived by the following inequality

$$
U \geq \mu(0)-\lambda^{*} B_{k}
$$

Note that the total volume of product $k$ can not be greater than $M$, i.e. $t_{k} \leq \frac{M}{v_{k} d_{k}}$. Therefore,

$$
\begin{aligned}
U \geq \mu(0)-\lambda^{*}\left(\sum_{i \neq k} \frac{1}{n_{i k}} \frac{r_{i} v_{k} d_{k} v_{i} d_{i}}{r_{i} v_{k} d_{k}+v_{i} d_{i}}\right)\left(\frac{M}{v_{k} d_{k}}\right), \\
\mu(0)-U \leq \lambda^{*}\left(\sum_{i \neq k} \frac{1}{n_{i k}} \frac{r_{i} v_{k} d_{k} v_{i} d_{i}}{r_{i} v_{k} d_{k}}\right)\left(\frac{M}{v_{k} d_{k}}\right) \\
\leq \lambda^{*}\left(\frac{1}{n_{i k}} v_{i} d_{i}+\sum_{j \neq i, k} v_{j} d_{j}\right)\left(\frac{M}{v_{k} d_{k}}\right), i \neq k .
\end{aligned}
$$

Hence,

$$
n_{i k} \leq \lambda^{*} v_{i} d_{i}\left[(\mu(0)-U)\left(\frac{v_{k} d_{k}}{M}\right)-\lambda^{*} \sum_{j \neq i, k} v_{j} d_{j}\right]^{-1}=u_{i k}, i \neq k
$$

\section{Algorithms and Computational Results}

Based on the theoretical results presented in the previous sections, we now propose a heuristic algorithm for solving the problem (1) - (2).

Algorithm 1:

Step 1: Solve (1) without capacity constraint (2) and obtain the Economic Order Quantity (EOQ) solution $s_{i}=0, t_{i}=\sqrt{2 c_{i} / h_{i} d_{i}}, i=1, \cdots, m$. If the EOQ solution satisfies (2), then it is optimal and stop. Otherwise, go to Step 2.

Step 2: Solve (26) with $B=0$ for the optimal Lagrangian multiplier $\lambda^{*}$ and let $U=\mu(0)$.

Step 3: For $k=1, \cdots, m$,perform Step 4 to Step 9.

Step 4: If a feasible solution of (1) - (2) with the objective value $U^{\prime}, \mu\left(B_{k}\right) \leq U^{\prime}<U$, is available, then $U$ is replaced by $U^{\prime}$. For each $i, i \neq k$, calculate the upper bound $u_{i k}$ of $n_{i k}$ according to $(27)$.

Step 5: For each $\left(\underline{n}_{i k}\right)_{i \neq k}, 1 \leq \underline{n}_{i k} \leq u_{i k}, i \neq k$, perform Step 6 to Step 8 .

Step 6: Solve $\left(a_{i}^{*}\right)_{i \neq k}$ and $\left(r_{i}^{*}\right)_{i \neq k}$ such that $\Phi$ is minimized. If (21) is satisfied with $\left(a_{i}\right)_{i \neq k}=$ $\left(a_{i}^{*}\right)_{i \neq k}$ and $\left(r_{i}\right)_{i \neq k}=\left(r_{i}^{*}\right)_{i \neq k}$, then solve (24) for given $\left(\underline{n}_{i k}\right)_{i \neq k}$ and go to Step 8. Otherwise, go to Step 7.

Step 7: Solve $\left(a_{i}^{*}\right)_{i \neq k}$ and $\left(r_{i}^{*}\right)_{i \neq k}$, such that $\Psi$ is minimized and solve (25) for given $\left(\underline{n}_{i k}\right)_{i \neq k}$. Step 8: If a feasible solution of (1) - (2) with the objective value $U^{\prime}, \mu\left(B_{k}\right) \leq U^{\prime}<U$, is obtained, then $U$ is replaced by $U^{\prime}$ and $u_{i k}, i \neq k$, are updated by using (27).

Step 9: Set $L_{k}=U$.

Step 10: Output the heuristic solution with the objective value $L^{*}=\min \left\{L_{k} \mid k=1, \cdots, m\right\}$.

A reasonable way to improve the current feasible solution $\left(t_{i}, s_{i}\right)$ is to search for a better solution in its neighborhood. In the following, we propose such an algorithm. 
Algorithm 2:

Step 1: Give a positive constant $\delta$ and positive integers $\zeta$ and $N . \delta / \zeta$ is the step size for the neighborhood search and $N$ is the maximum number of iterations allowed in the algorithm. Set $t_{i}^{*}=t_{i}, s_{i}^{*}=s_{i}, i=1, \cdots, m$ and $I=0$ ( $I$ is a counter of the number of iterations.)

Step 2: Set $I=I+1$. If $I \leq N$, perform Step 3 and Step 4 for all the combinations of integer values $\phi_{1}, \cdots, \phi_{m}$ such that $\left|\phi_{i}\right| \leq \zeta, i=1, \cdots, m$. Otherwise, go to Step 5 .

Step 3: Set $t_{i}=t_{i}^{*}+\phi_{i}(\delta / \zeta), i=1, \cdots, m$. If the objective value $Z$ associated with $\left(t_{i}\right)$ is less than that associated with $\left(t_{i}^{*}\right)$, then go to Step 4 . Otherwise, check the next combination of integer values $\phi_{1}, \cdots, \phi_{m}$.

Step 4: Calculate the $n_{i j}$ 's and solve

$$
\min _{k} \min _{\left(r_{i}\right)}\left\{\left(v_{k} d_{k}+\sum_{i \neq k} \frac{n_{k i}}{n_{i k}} v_{i} d_{i}-\sum_{i \neq k} \frac{1}{n_{i k}} \frac{r_{i} v_{k} d_{k} v_{i} d_{i}}{r_{i} v_{k} d_{k}+v_{i} d_{i}}\right) t_{k} \mid \sum_{i \neq k} r_{i}=1, r_{i} \geq 0\right\} .
$$

If the optimal value is not greater than $M$ for $k=k_{0}$ and $\left(r_{i}\right)_{i \neq k_{0}}=\left(r_{i}^{*}\right)_{i \neq k_{0}}$, then set $s_{k_{0}}^{*}=0, s_{i}^{*}=\left(t_{k_{0}} / n_{i k_{0}}\right)\left[v_{i} d_{i} /\left(r_{i}^{*} v_{k_{0}} d_{k_{0}}+v_{i} d_{i}\right)\right], i=1, \cdots, k_{0}-1, k_{0}+1, \cdots, m$ and $t_{i}^{*}=t_{i}, i=1, \cdots, m$, and go to Step 2 . Otherwise, check the next combination of integer values $\phi_{1}, \cdots, \phi_{m}$.

Step 5: Output the solution $\left(t_{i}^{*}\right)$ and $\left(s_{i}^{*}\right), i=1, \cdots, m$.

In the following, we consider three-product problems with various warehouse capacities. These problems are solved by three different approaches and their computational results are shown in Table 3.

Table 1. A three-product problem.

\begin{tabular}{rrrrl}
\hline$i$ & 1 & 2 & 3 & \\
\hline$c_{i}$ & 50 & 50 & 50 & $m=3$ \\
$h_{i}$ & 10 & 4 & 16 & $M=15000$ \\
$d_{i}$ & 1000 & 1000 & 2000 & \\
$v_{i}$ & 50 & 20 & 80 & \\
\hline
\end{tabular}

Table 2. The solutions of EOQ method and the proposed algorithm.

\begin{tabular}{cccccc}
\hline $\begin{array}{c}\text { Solution } \\
\text { Method }\end{array}$ & $t_{1}$ & $t_{2}$ & $t_{3}$ & $\begin{array}{c}\text { Total } \\
\text { Cost }\end{array}$ & $\begin{array}{c}\text { Maximum } \\
\text { Stock Level }\end{array}$ \\
\hline EOQ & 0.1000 & 0.1581 & 0.0559 & 3421.11 & 17106 \\
Algorithm 1 & 0.1106 & 0.1659 & 0.0553 & 3427.20 & 15000 \\
& $s_{1}=0.0000$ & $s_{2}=5.5 \times 10^{-9}$ & \multicolumn{2}{c}{$s_{3}=0.0385$} \\
\hline
\end{tabular}


Table 3. Solution inventory costs from four algorithms.

\begin{tabular}{|c|c|c|c|c|c|c|}
\hline $\begin{array}{c}\text { Warehouse } \\
\text { Capacity } \\
\text { M }\end{array}$ & $\begin{array}{l}\text { Lagrangian } \\
\text { Multiplier } \\
\text { Method (A) }\end{array}$ & $\begin{array}{c}\text { Fixed Cycle } \\
\text { Method } \\
\text { (B) }\end{array}$ & $\begin{array}{c}\text { Algorithm } 1 \\
\text { (C) }\end{array}$ & $\begin{array}{l}\text { Algorithm 1\& } \\
\text { Neighborhood } \\
\text { Search }\end{array}$ & $\begin{array}{c}(\mathrm{C}) /(\mathrm{A}) \\
100 \%\end{array}$ & $\begin{array}{c}(\mathrm{C}) /(\mathrm{B}) \\
100 \%\end{array}$ \\
\hline 100 & 292644.04 & 265447.78 & 249574.47 & 249574.47 & 85.28 & 94.02 \\
\hline 200 & 146337.02 & 132743.39 & 124804.86 & 124804.86 & 85.29 & 94.02 \\
\hline 300 & 97574.68 & 88517.25 & 83222.83 & 83222.83 & 85.29 & 94.02 \\
\hline 400 & 73198.51 & 66410.69 & 62437.69 & 62437.69 & 85.30 & 94.02 \\
\hline 500 & 58576.81 & 53151.94 & 49971.31 & 49971.31 & 85.31 & 94.02 \\
\hline 600 & 48832.34 & 44317.12 & 41664.31 & 41664.31 & 85.32 & 94.01 \\
\hline 700 & 41874.86 & 38010.24 & 35734.09 & 35734.09 & 85.34 & 94.01 \\
\hline 800 & 36659.25 & 33283.33 & 31289.37 & 31289.37 & 85.35 & 94.01 \\
\hline 900 & 32604.89 & 29609.73 & 27834.98 & 27834.98 & 85.37 & 94.01 \\
\hline 1000 & 29363.40 & 26673.45 & 25073.81 & 25073.81 & 85.39 & 94.00 \\
\hline 2000 & 14831.70 & 13531.69 & 12713.21 & 12713.21 & 85.72 & 93.95 \\
\hline 3000 & 10054.47 & 9237.75 & 8671.37 & 8671.37 & 86.24 & 93.87 \\
\hline 4000 & 7715.85 & 7155.77 & 6709.22 & 6709.22 & 86.95 & 93.75 \\
\hline 5000 & 6352.69 & 5958.57 & 5578.96 & 5578.96 & 87.82 & 93.63 \\
\hline 6000 & 5477.23 & 5203.77 & 4864.61 & 4864.61 & 88.82 & 93.48 \\
\hline 7000 & 4880.49 & 4701.75 & 4387.95 & 4387.95 & 89.91 & 93.33 \\
\hline 8000 & 4457.93 & 4357.73 & 4059.85 & 4057.85 & 91.07 & 93.16 \\
\hline 9000 & 4151.49 & 4119.05 & 3830.77 & 3830.77 & 92.27 & 93.00 \\
\hline 10000 & 3926.34 & 3954.10 & 3666.44 & 3666.44 & 93.38 & 92.73 \\
\hline 11000 & 3760.31 & 3842.77 & 3558.34 & 3558.34 & 94.63 & 92.59 \\
\hline 12000 & 3638.62 & 3771.66 & 3487.85 & 3487.85 & 95.86 & 92.48 \\
\hline 13000 & 3551.03 & 3731.48 & 3448.28 & 3448.28 & 97.11 & 92.41 \\
\hline 14000 & 3490.24 & 3715.61 & 3428.29 & 3428.29 & 98.23 & 92.27 \\
\hline 15000 & 3450.89 & 3714.84 & 3427.20 & 3427.20 & 99.94 & 92.26 \\
\hline 16000 & 3428.96 & 3714.84 & 3427.00 & 3427.00 & 99.94 & 92.25 \\
\hline 17000 & 3421.38 & 3714.84 & 3421.38 & 3421.36 & 100.00 & 92.10 \\
\hline 17106 & 3421.11 & 3714.84 & 3421.11 & 3421.11 & 100.00 & 92.09 \\
\hline
\end{tabular}

In Table 2 and Table 3 , we note that if $M \geq 17106$, then the problem can be solved without capacity constraint. In this case, both the EOQ method and the Lagrangian multiplier method yield the optimal solution. It can be seen that as the restriction on the warehouse capacity gets tighter, the fixed cycle method is often better that the Lagrangian multiplier method. However, for some problem instances the fixed cycle method may never generate an optimal solution for any given $M>0$. In fact, these two methods do not solve the problem in an optimal way. Thus, when faced with the warehouse capacity restriction, it is essential to use the staggering policy and allow different products to have different order intervals. In Table 3, we also note that the proposed algorithm is significantly better than the two methods mentioned above. 


\section{Acknowledgements}

The authors would like to thank the Editor and two anonymous referees for their helpful comments and suggestions.

\section{References}

[1] C.-C. Chen, M.-S. Chern and K.-C. Lin: Capacitated Multiple Products Inventory System, Recent Developments in Production Research (Editor: Anil Mital), Elsevier Science Publishers B.V., Amsterdam (1988), 194-199.

[2] S.K. Goyal: A Note on 'Multi-Product Inventory Situations with one Restriction', Journal of the Operational Research Society, Vol. 29, (1978), 269-271.

[3] G. Hadley and J.M. Whitin, Analysis of Inventory System, Prentice-Hall, Englewood Cliffs, N.J., 1963.

[4] R. Hartley and L.C. Thomas: The Deterministic, Two-Product, Inventory System with Capacity Constraint, Journal of the Operational Research Society, Vol. 33 (1982), 10131020.

[5] E. Horowitz and S. Sahni, Fundamentals of Computer Algorithms, Computer Science Press, Rockville, MD, 1978.

[6] E. Page and K.J. Paul: Multi-Product Inventory Situations with one Restriction, Operational Research Quarterly, Vol. 27 (1976), 815-834.

[7] H. Pirkul and O.A. Aras: Capacitated Multiple Item Ordering Problem with Quantity Discount, IIE Transactions, Vol. 17 (1985), 206-211.

[8] H.J. Rosenblatt: Multi-Item Inventory System with Budgetary Constraint: A Comparison Between the Lagrangian and Fixed Cycle Approach, International Journal of Production Research, Vol. 19 (1981), 331-339.

[9] L.C. Thomas and R. Hartley: An Algorithm for the Limited Capacity Inventory Problem with Staggering, Journal of the Operational Research Society, Vol. 34 (1983), 81-85.

[10] K. Zoller: Deterministic Multi-Item Inventory Systems with Limited Capacity, Management Science, Vol. 24 (1977), 451-455.

Maw-Sheng Chern

Dept. of Industrial Engineering

National Tsing Hua University

Hsinchu (30043)

Taiwan 